ÁREA ABIERTA. Vol.13. n³. Noviembre 2013

http://dx.doi.org/10.5209/rev_ARAB.2013.v34.n3.43350

Referencia: AA34-3.1211.172

"SIGLO XXI Y MONARQUÍA. PROPUESTAS PARA DINAMIZAR

LA CARACTERIZACIÓN INFORMATIVA DEL REY JUAN CARLOS I"

AUTOR: Dr. Daniel BARREDO IBÁÑNEZ

Escuela Superior Técnica de Chimborazo. Ecuador

\title{
Siglo XXI y monarquía. Propuestas para dinamizar la caracterización informativa del rey Juan Carlos I
}

21 st Century and the Monarchy. Proposals to Dynamize

the Informative Characterization of King Juan Carlos I 


\section{RESUMEN:}

En un contexto caracterizado por la participación ciudadana y el refuerzo de los canales interactivos de las instituciones, la Casa Real Española se percibe como un entorno informativo altamente restrictivo: opaco, tabú y direccional. La caída de la popularidad de la monarquía obliga a la institución a replantearse el espacio relacional que comparte con los medios de comunicación que son, ante todo, los constructores de los significados simbólicos en los que se sustenta su reputación. A través del consenso de unos expertos en comunicación institucional, este trabajo ofrece algunas pautas para dinamizar el discurso informativo de la Casa del Rey. Dos ejes fundamentales identifican casi todas las recomendaciones propuestas por el grupo para reforzar el prestigio de esta institución en crisis: interactividad con la sociedad y transparencia de la gestión comunicacional.

\section{Palabras clave:}

Juan Carlos I, comunicación institucional, Casa Real, interactividad, método Delphi.

\section{ABSTRACT:}

In a context characterized by the citizen participation and the reinforcement of the institutions' interactive channels, the Spanish Royal Family is seen as a highly-restrictive informative environment: opaque, directional and full of taboos. The fall of the popularity of the monarchy makes the institution reconsider the relational space it shares with the mass media which are, above all, the builders of the symbolic meanings which support its reputation. Based on the agreement of a group of institutional communication experts, this paper provides several guidelines to dynamize the Royal Family's informative speech. Two core axes identify almost every recommendation proposed by the group in order to reinforce the prestige of this institution, which is in a crisis: interactivity with the society and the communicative management transparency.

\section{Key words:}

Juan Carlos I, Institutional communication, Royal Family, interactivity, Delphi approach/method. 


\section{Introducción}

La monarquía española es uno de los entornos informativos más restrictivos para los periodistas de la democracia actual. Para empezar porque el rey Juan Carlos, a diferencia de otras figuras públicas, no suele responder a ningún tipo de pregunta. Los comunicadores cuentan con un tiempo estipulado para hacer su trabajo; sólo a través de la rotura del estricto protocolo pueden conseguir un hecho noticioso al margen de la declaración oficial². Pero es que además los periodistas se enfrentan a una institución que en ocasiones se asocia al secuestro de publicaciones ${ }^{3}$, a un blindaje a través de los artículos 490 y 491 del Código Penal español ${ }^{4}$, a un contexto histórico en que los principales medios establecieron el ensalzamiento común de los atributos positivos del monarca ${ }^{5}$, y a un aparato legal que incluso el Tribunal Europeo de Derechos Humanos ha criticado por la "sobreprotección" del sistema monárquico ${ }^{6}$. No extraña, por tanto, que la Casa Real haya sido clasificada entre los principales tabús de los periodistas españoles en el siglo XXI7.

En octubre de 2011 la monarquía suspendió en el nivel de confianza popular recogido por el barómetro del Centro de Investigaciones Sociológicas ${ }^{8}$. Y eso a pesar de que fue en noviembre del mismo año, un mes después, cuando se destapó en los medios de comunicación el llamado caso Urdangarin a través del cual el yerno del monarca fue imputado por malversación de caudales públicos9. La disociación de valores como la honorabilidad, como consecuencia de tener a uno de sus miembros sentado en el banquillo de los acusados, ha invertido la significación del patrimonio simbólico de la institución y ha aumentado la brecha en la reputación de sus representantes, algo que ha comenzado a preocupar seriamente a los responsables de comunicación de la Casa Real. Incluso el Rey en su discurso navideño de 2011 abordó directamente la paulatina falta de credibilidad o distanciamiento entre la sociedad y el discurso de su Casa:

\footnotetext{
${ }^{1}$ Parte de este trabajo se expuso en el grupo de trabajo "Reputación de las instituciones: retos, propuestas y estrategias" del XXVII Congreso Internacional de Comunicación "Generar confianza: la comunicación de instituciones en tiempos de crisis", celebrado los días 22 y 23 de marzo de 2012 en la Universidad de Navarra (España).

${ }^{2}$ Cfr. BARREDO IBÁÑEZ, D., El tabú Real. La imagen de una monarquía en crisis, Córdoba, Berenice, 2013.

${ }^{3}$ Cfr. RAMOS, F., "El secuestro de El Jueves y las injurias periodísticas a la corona, un injustificable ataque a la libertad de expresión", ÁMBITOS, núm. 16, 2007, pp. 151-186.

${ }^{4}$ Un delito por injurias al Rey puede ser castigado con penas de prisión de entre seis meses y dos años, si se considera grave o bien, en caso de no ser grave, con una multa de seis a doce meses.

${ }^{5}$ Cfr. ZUGASTI, R., La forja de una complicidad. Monarquía y Prensa en la Transición española (1975 - 1978), Fragua, Madrid, 2007.

${ }^{6}$ EUROPEAN COURT OF HUMAN RIGHTS, "An elected representative's conviction for causing serious insult to the King of Spain was contrary to his freedom of expression", Press release by the Registrar of the Court, núm. 215 , 2011 , P. 3.

${ }^{7}$ BARREDO IBÁÑEZ, D., "Tabús informativos individuales y organizacionales del periodismo español en el siglo XXI: un estudio Delphi", Fonseca, Journal of Communication, núm. 3, 2011, p. 114.

8 CIS, "Barómetro de octubre", núm. 2914, 2011, p. 9, obtenido el 10 de enero de 2012 de http://www.cis.es/cis/export/sites/default/-Archivos/Marginales/2900_2919/2914/Es2914.pdf.

9 MANRESA, A., "El juez levanta el secreto e imputa a Iñaki Urdangarin", El País, 29-12-2011, obtenido el 10 de enero de 2012 de http://politica.elpais.com/politica/201 1/12/29/actualidad/1325146927_909959.html.
} 
Junto a la crisis económica, me preocupa también enormemente la desconfianza que parece estar extendiéndose en algunos sectores de la opinión pública respecto a la credibilidad y prestigio de algunas de nuestras instituciones. Necesitamos rigor, seriedad y ejemplaridad en todos los sentidos. Todos, sobre todo las personas con responsabilidades públicas, tenemos el deber de observar un comportamiento adecuado, un comportamiento ejemplar ${ }^{10}$.

Con el fin de reorientar el prestigio institucional la Casa Real ha puesto en marcha una serie de medidas entre las que destaca la publicación del desglose de su presupuesto, un hecho histórico en las casi cuatro décadas de reinado ${ }^{11}$.

Este trabajo se enmarca en este contexto de cambio o de reconfiguración del modelo comunicacional por el que optó desde sus inicios la institución monárquica. Nos hemos propuesto responder a las siguientes preguntas de investigación:

PI1: ¿Cuál es la caracterización informativa de la Casa Real en relación con el funcionamiento de otras instituciones representativas tales como la presidencia del Gobierno?

PI2: ¿̇Es conveniente, en términos de prestigio, el nivel restrictivo adoptado por la institución?

PI3: ¿Qué nuevos canales podrían reconfigurar el espacio relacional entre la Casa del Rey y los medios de comunicación españoles?

\section{Objetivos}

El interés del siguiente trabajo se razona en función de los siguientes objetivos:

1. Exploratorios. Describir un fenómeno inexplorado todavía: el de las restricciones inherentes a la Casa Real. Este proyecto pretende introducir las coordenadas básicas a partir de las cuales va a desarrollarse una unidad analítica mayor ${ }^{12}$.

2. Procedimentales. Proponer unos procedimientos metodológicos novedosos para definir el funcionamiento de los entornos informativos institucionales.

\footnotetext{
${ }^{10}$ DE BORBÓN, J. C., "Mensaje de Navidad de Su Majestad el Rey", Casa de Su Majestad el Rey, 24-12-2011, obtenido el 10 de enero de 2012 de http://www.casareal.es/noticias/news/20111224_mensaje_navidad-idesidweb.html.

${ }^{11}$ El desglose presupuestario es accesible a todos los ciudadanos a través de la página web oficial de la Casa Real. Cfr. "Presupuesto anual", Casa de Su Majestad el Rey, (sin fecha), obtenido el 10 de enero de 2012 de http://www.casareal.es/laCasa/presupuesto-ides-idweb.html.

${ }^{12}$ Es este uno de los estudios preliminares que realizamos para desarrollar la tesis doctoral "El tabú de la expresividad real. Análisis del tratamiento informativo del rey Juan Carlos I en ABC.es y ELPAís.com (2009 2011), defendida el 10/01/2013, en la Universidad de Málaga, con la máxima calificación.
} 
3. Socioinstitucionales. Mediar entre las decisiones de los representantes institucionales y los representados sociales. En ese sentido las nuevas medidas aprobadas por la Casa Real denotan una clara disposición interna por reubicar su discurso en el contexto actual. Esas medidas no han de entenderse como un acto arbitrario de la institución, sino más bien como parte del creciente debate social en el que está inmersa la monarquía.

\section{Metodología}

\subsection{Cuestionario}

El carácter inexplorado del proyecto aconsejaba, como acercamiento preliminar, la utilización de una metodología Delphi. El análisis a través de este método ha sido ensalzado por su capacidad para anticipar tendencias sociopolíticas pero también por su facilidad para generar nuevos escenarios de estudio ${ }^{13}$. Una prospección basada en esta metodología busca sobre todo construir un nivel aceptable de consenso entre un panel determinado de individuos ${ }^{14}$. Son cuatro las características de un proyecto Delphi: proceso iterativo, anonimato de los participantes, feedback controlado y respuesta estadística del grupo $^{15}$. En este proyecto en primer lugar se diseñó un instrumento que contenía 39 ítems repartidos en siete cuestiones articuladas en función de las preguntas de investigación que encabezan este trabajo. Las siete cuestiones a su vez atendían a tres bloques temáticos íntimamente relacionados pero cada uno de ellos dotado con unos objetivos concretos. Los siguientes esquemas (figuras $n^{\circ} 1,2$ y 3) resumen la composición de las variables junto con sus objetivos específicos dentro de los tres bloques temáticos enunciados; como se verá a continuación la numeración de los interrogantes se reordenó aleatoriamente en el cuestionario para evitar el denominado "efecto halo" o sesgo cognitivo.

\begin{tabular}{|l|l|l|}
\hline \multicolumn{2}{|c|}{ Figura n 1. Primer bloque. Descripción de la situación informativa actual } \\
\hline Pregunta 1 & Objetivos & Pregunta 4 \\
\hline $\begin{array}{l}\text { Define la caracterización } \\
\text { de las informaciones sobre } \\
\text { el Rey }\end{array}$ & $\begin{array}{l}\text { - Describir la tendencia individual } \\
\text { de cada experto sobre los dos } \\
\text { temas propuestos } \\
\text { - Comparar las diferencias entre } \\
\text { las tendencias }\end{array}$ & $\begin{array}{l}\text { Define la caracterización de } \\
\text { las informaciones sobre el } \\
\text { Presidente del Gobierno }\end{array}$ \\
\hline
\end{tabular}

Fuente: elaboración propia

\footnotetext{
${ }^{13}$ Cfr. TEZANOS, J. F., "El método Delphi y la anticipación de escenarios y tendencias socio - políticas", Sistema: revista de ciencias sociales, núm. 213, 2009, pp. 83-104.

${ }^{14}$ Cfr. LINSTONE, H. A. y TUROFF, M., "Introduction", en LINSTONE, H. A. y TUROFF, M. (eds.), The Delphi method: Techniques and applications, Addison-Wesley Publishing Company, Reading, 1975, pp. 3-12; HSU, Chia-Chien y SANDFORD, B. A., "The Delphi Technique: Making Sense of Consensus", Practical Assesment Research and Evaluation, núm. 12, vol. 10, 2007, obtenido el 10 de enero de 2012 de http://pareonline.net/pdf/v12n10.pdf.

15 LANDETA, J., El método Delphi. Una técnica de previsión para la certidumbre, Ariel, Barcelona, 1999, pp. 42 44.
} 


\begin{tabular}{|l|l|l|}
\hline \multicolumn{2}{|c|}{ Figura $\mathbf{n}^{\circ}$ 2. Segundo bloque. Valoraciones íntimas } \\
\hline Pregunta 2 & Objetivos & Pregunta 6 \\
\hline $\begin{array}{l}\text { Cuestiona la necesidad de } \\
\text { un incremento de la } \\
\text { transparencia informativa } \\
\text { sobre el Rey }\end{array}$ & $\begin{array}{l}\text { - Averiguar la valoración íntima } \\
\text { de los entrevistados }\end{array}$ & $\begin{array}{l}\text { Cuestiona la validez del } \\
\text { blindaje informativo sobre } \\
\text { determinados aspectos }\end{array}$ \\
\hline
\end{tabular}

Fuente: elaboración propia

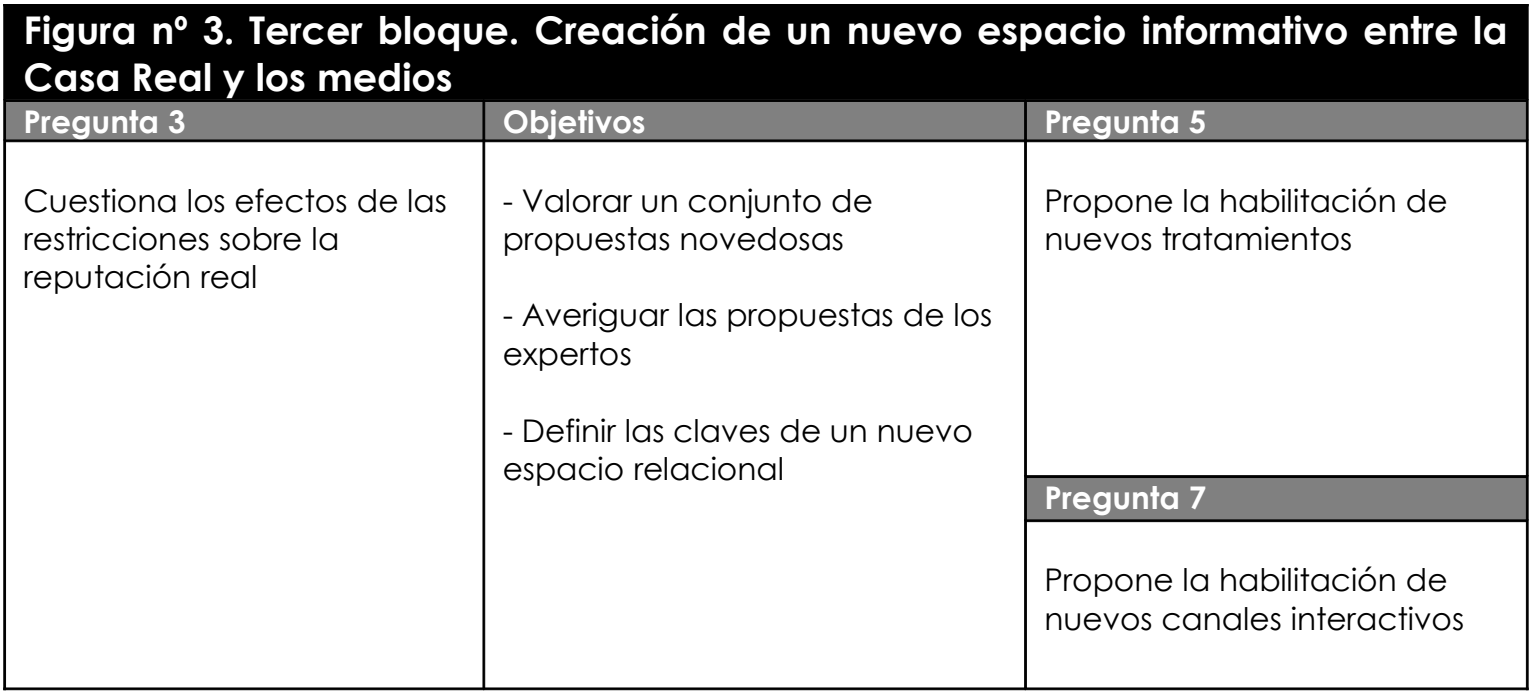

Fuente: elaboración propia

Las respuestas del primero de los bloques se cifraron mediante una escala basada en el método Diferencial Semántico (DS) de Osgood, Suci y Tannenbaum ${ }^{16}$. Es este uno de los métodos más utilizados en la investigación social debido a su eficacia ${ }^{17}$. Siguiendo el modelo origina ${ }^{18}$ diseñamos una escala inicial de 30 adjetivos divididos por sus características en evaluativos, de actividad y de potencia. Después de realizar una prueba de confiabilidad durante la fase pretest redujimos la escala final a 10 adjetivos bipolares que eran los que presentaban una menor oscilación en sus varianzas. Como puede observarse a continuación un 30\% $(n=6)$ de los adjetivos incluidos "direccionalinteractiva, disuasiva-persuasiva, tendenciosa-equilibrada" define características exclusivas del mensaje informativo, no extrapolables a otros contextos discursivos debido a su alta especialización.

\footnotetext{
${ }^{16}$ Cfr. OSGOOD, C. E., SUCl, G. J. y TANNENBAUM, P. H., The measurement of meaning, University of Illinois Press, Illinois, 1957.

17 ARCE FERNÁNDEZ, C., STEM, D. E., ANDRADE FERNÁNDEZ, E. M. y SEOANE PESQUEIRA, G., "Comparación de escalas de respuesta para la medición de las actitudes", Psicothema, núm. 9, vol. 3, 1997, p. 541.

${ }^{18}$ Cfr. OSGOOD, C. E., SUCl, G. J. y TANNENBAUM, P. H., op. cit.
} 


\begin{tabular}{|l|l|l|}
\hline \multicolumn{2}{|c|}{ Figura nं 4. Escala de los adjetivos bipolares } \\
utillizados en el cuestionario
\end{tabular}

Fuente: elaboración propia

En el cuadro anterior puede apreciarse un mayor número de adjetivos catalogados como evaluativos frente a esos otros clasificados como de actividad o de potencia. Este desnivel es frecuente en los estudios que aplican el DS ya que los adjetivos evaluativos describen los factores más próximos a la actitud, que es uno de los objetos más importantes de este tipo de medición ${ }^{19}$. Los adjetivos bipolares se separaron en el cuestionario por siete tramos codificados por letras "a", "b", "c", "d", "e", "f", "g" en vez de números, porque los números tienden a favorecer el sesgo cognitivo en función del valor representado ${ }^{20}$. En las instrucciones que encabezaban al instrumento se especificó que la letra "d" era el tramo neutro o intermedio, y se pidió a los entrevistados que salvo en casos estrictamente necesarios no puntuaran los diferentes ítems a través de este tramo ya que no aportaba una información relevante. Tal y como es habitual en las respuestas confeccionadas mediante el DS el grado de proximidad de las letras con respecto de los adjetivos señalaba la coincidencia de la opinión del entrevistado. Así, por ejemplo, las letras "a" y "g" eran las posiciones más distanciadas, más extremas, por lo que representaban un mayor grado de coincidencia con cada uno de los adjetivos. Una vez procesadas las respuestas las letras se recodificaron reasignando a la escala unos valores numéricos correlativos de 1 a 7 . En este apartado el alto nivel de consenso se estableció en una desviación típica igual o superior a 2.5 puntos, el nivel de consenso medio se fijó entre 2.51 y 3 puntos y el desacuerdo se identificó a partir de una desviación superior a los 3 puntos.

Para la elaboración del bloque temático esquematizado en la figura $n^{\circ} 2$ se diseñó un experimento inspirado en las experiencias del Instituto Demoscópico Allensbach ${ }^{21}$, y mejorado a partir de un proyecto de investigación anterior ${ }^{22}$. El instrumento describía una discusión entre dos periodistas durante un viaje en avión. Cada uno de ellos mantenía una opinión extrema claramente diferenciada por los enunciados. El entrevistado tenía que señalar con cuál de los dos contertulios se identificaba más; de esta manera se favorecía la revelación de las valoraciones íntimas de los encuestados sin cargarles con el peso

\footnotetext{
19 BECOÑA IGLESIAS, E., "El Diferencial Semántico en la medición de actitudes: adjetivos versus conceptos, Revista de Psicología General y Aplicada, núm. 43, vol. 3, 1990, p. 302.

${ }^{20}$ Cfr. NADELSTICHER, A., Técnicas para la construcción de cuestionarios de actitudes y opción múltiple, Instituto Nacional de Ciencias Penales, México, 1983.

${ }^{21}$ Cfr. NOELLE - NEUMANN, E., La espiral del silencio. Opinión pública: nuestra piel social, Paidós, Barcelona, 1995.

${ }^{22}$ BARREDO IBÁÑEZ, D., op. cit., pp. 118-119, 2011.
} 
ideológico de la tendencia mayoritaria, o sin enfrentarles con una pregunta directa que hubiera podido filtrar algunos de los elementos que detienen el desarrollo social tales como la timidez. Para forzar las valoraciones se eliminó el tramo intermedio y se ofrecieron unas respuestas dicotómicas de alternativa simple. En el primer coloquio se planteaba la necesidad de una mayor transparencia sobre los asuntos de la Casa Real. En el segundo se cuestionaba la necesidad del blindaje informativo alrededor de determinados asuntos del monarca. Para las respuestas dicotómicas el alto nivel de consenso se estableció en un porcentaje igual o superior al $70 \%$ de las respuestas, el nivel medio de consenso se cifró en el $60 \%$ y el desacuerdo se fijó a partir de un porcentaje inferior al $59 \%$ de las respuestas.

Finalmente el tercer bloque de preguntas se construyó mediante una pregunta mixta de alternativa múltiple y dos preguntas mixtas con escalas tipo Likert de 1 a 5 puntos. Estas dos últimas preguntas solicitaban además a cada uno de los entrevistados la aportación individual de dos ítems no recogidos en la lista ofrecida, de manera que en la segunda ronda los participantes pudieron puntuar sus propias respuestas. En este tercer bloque el alto nivel de consenso se estableció para aquellas respuestas que registraban una desviación típica inferior a 1.5 puntos, el nivel medio de consenso se fijó entre 1.51 puntos y 2 puntos y el desacuerdo se contabilizó a partir de una desviación superior a los 2 puntos.

\subsection{Pretest}

Una vez concluido el cuestionario se realizó una fase de simulación o pretest con el fin de determinar o verificar:

a) La fiabilidad y la selección de los adjetivos bipolares presentes en el primer bloque de preguntas del instrumento

b) La claridad de los interrogantes y de las respuestas

c) La precisión de las respuestas a las preguntas de investigación

\subsection{Muestra}

Dado el carácter introductorio de este estudio se optó por confeccionar una muestra integrada por expertos altamente especializados. Para la elección del panel se tuvieron en cuenta todos o la mayor parte de los siguientes parámetros:

a) La publicación de libros o artículos relevantes en materia de comunicación institucional o protocolo en el contexto científico español.

b) Una trayectoria consolidada dentro del asesoramiento en cuestiones relativas al prestigio o a la identidad institucionales.

Asimismo se procuró, en la medida de lo posible, mantener un equilibrio entre los siguientes aspectos: 

a) La edad de los integrantes
b) La adscripción ideológica
c) El género
d) La región histórica en que desarrollan sus actividades profesionales

\subsubsection{Integrantes de la muestra}

El número de integrantes de un panel de expertos es siempre un proceso que depende en buena medida de los criterios del investigador pero también de factores externos tales como la disponibilidad de los entrevistados ${ }^{23}$. En los proyectos basados en la metodología Delphi se ha fijado en 3 personas el tamaño mínimo de la muestra y en 171 el número máximo de integrantes ${ }^{24}$. El tamaño está inextricablemente ligado al objetivo exploratorio de estos proyectos: más que averiguar un conjunto relativamente extenso de opiniones se trata de consolidar la opinión de un grupo que comparte la alta especialización en una materia $^{25}$. Para este estudio se escogieron inicialmente nueve expertos de los cuales un experto no respondió a la solicitud de participación, un experto declinó participar porque afirmó que carecía de información sobre el fenómeno propuesto y un experto declinó participar porque alegó que sus respuestas podían desprestigiar a su centro de trabajo. En total seis expertos aceptaron formar parte del proyecto. Durante la fase de desarrollo sus nombres se mantuvieron en el anonimato con el fin de evitar cualquier tipo de injerencia. Los integrantes del panel fueron, por orden alfabético:

Dra. Ana Almansa. Universidad de Málaga.

Dr. Fernando Martín. Universidad San Pablo CEU de Madrid.

Dr. Justo Villafañe. Universidad Complutense de Madrid.

Dr. Luis Fernando Ramos. Universidad de Vigo.

Dr. Ramón Zallo. Universidad del País Vasco.

Dra. Yolanda Martínez Solana. Universidad Complutense de Madrid.

\subsubsection{Número de rondas}

El número de rondas de un proyecto basado en la metodología Delphi depende en buena medida del número de expertos que integran la muestra como también del grado de desacuerdo que se manifiesta entre ellos. En este proyecto la alta especialización compartida entre los integrantes preveía una baja desviación. En un principio se estimó que harían falta dos rondas para conseguir una opinión grupal relativamente estable. La primera de ellas se desarrolló durante el mes de diciembre de 2011 . La segunda abarcó las tres primeras semanas de enero de 2012.

\footnotetext{
${ }^{23}$ Cfr. LANDETA, J., op. cit.

${ }^{24}$ Cfr. SKULMOSKI, G., HARTMAN, F. y KRAHN, J., "The Delphi Method for Graduate Research", Journal of Information Technology Education, núm. 6, 2007, pp. 1-21.

${ }^{25}$ Cfr. HSU, C.-C. y SANDFORD, B. A., op. cit.
} 


\subsubsection{Envío del cuestionario}

Tanto en la primera como en la segunda rondas se utilizó el correo electrónico como forma de contacto con los entrevistados. Esta tecnología permite varias ventajas con respecto de otros canales tradicionales (correo postal, teléfono), como son el ahorro de tiempo, la accesibilidad, la rapidez de las respuestas o la reducción de los costes de la investigación ${ }^{26}$. En la primera ronda el cuestionario se envió a cada uno de los expertos junto con una carta estandarizada, una hoja de referencias académicas, el currículo del investigador y una explicación personalizada de los motivos por los que se había seleccionado a cada integrante. En la segunda ronda se remitió otra vez el cuestionario pero esta vez incluía como novedad la media de las respuestas grupales, las respuestas individuales de la primera ronda y una hoja de instrucciones en la que se advertía a los entrevistados de que las respuestas de la segunda ronda serían consideradas como definitivas. Asimismo en la segunda ronda se adjuntaron 11 nuevos ítems que recogían las aportaciones efectuadas por los expertos en la primera ronda a través de los apartados interactivos habilitados en las preguntas 5 y 7 del cuestionario.

\section{Resultados}

\subsection{Primer bloque: Descripción de la situación informativa actual}

En primer lugar nos fijamos como objetivo la descripción de la caracterización informativa de las noticias protagonizadas por el rey Juan Carlos I en los medios de comunicación españoles. La opinión conjunta de los integrantes del grupo, a pesar de las diferencias de edad, de región, de residencia, de tendencia ideológica o de los siete tramos de la escala en ninguna de las elecciones bipolares rebasó una desviación típica superior a los 2.5 puntos. Es decir las respuestas que ofrecemos en la siguiente tabla (figura $n^{\circ}$ 5) presentan un alto nivel de consenso grupal ${ }^{27}$.

\footnotetext{
${ }^{26}$ Cfr. YOUNG, S. J. y JAMIESON, L. M., "Delivery methodology of the Delphi: a comparison of two approaches", Journal of Park and Recreation Administration, núm. 19, vol. 1, 2001, pp. 42-58.

${ }^{27}$ Esta tabla recoge la clasificación de los adjetivos en función de las frecuencias según las medias grupales. Para conocer detalladamente los resultados del instrumento se adjuntan todos los datos en el Anexo 1 de este trabajo.
} 


\section{Figura $n^{\bullet}$ 5. Caracterización de la información protagonizada por el rey Juan Carlos en los medios de comunicación españoles}

\begin{tabular}{|c|c|c|c|c|c|c|}
\hline \multicolumn{3}{|c|}{ Espectro negativo } & \multirow[t]{2}{*}{ Espectro neutro } & \multicolumn{3}{|c|}{ Espectro positivo } \\
\hline $\begin{array}{l}\text { Extremada- } \\
\text { mente }\end{array}$ & Bastante & Poco & & Poco & Bastante & $\begin{array}{l}\text { Extremada- } \\
\text { mente }\end{array}$ \\
\hline & & & $\begin{array}{l}\text { Ni escasa } \\
\text { ni abundante }\end{array}$ & & & \\
\hline & & Aburrida & & & & \\
\hline & & Obsoleta & & & & \\
\hline & & Insignificante & & & & \\
\hline & Opaca & & & & & \\
\hline & & Inútil & & & & \\
\hline & & Tendenciosa & & & & \\
\hline & Tabú & & & & & \\
\hline & Direccional & & & & & \\
\hline & & & $\begin{array}{l}\text { Ni disuasiva } \\
\text { ni persuasiva }\end{array}$ & & & \\
\hline
\end{tabular}

Fuente: elaboración propia

La clasificación de los adjetivos dentro de las tres valencias o espectros significativos (negativo, neutro, positivo), se efectuó solamente a posteriori. Durante la fase de desarrollo los entrevistados no recibieron más elementos informativos aparte de los tipificados de forma expresa en la descripción metodológica de este trabajo. Según puede observarse en la figura $n^{\circ} 5$ la opinión grupal situó un elevado número de adjetivos dentro del espectro negativo $(n=8)$, posicionó dos adjetivos en el terreno neutro y finalmente no emplazó ningún adjetivo dentro del espectro positivo. Con el fin de verificar la validez de esta descripción decidimos repetir el experimento con el mismo procedimiento y con la misma escala de adjetivos bipolares aunque en esta ocasión desplazamos el sujeto institucional analizado desde el rey Juan Carlos I al Presidente del Gobierno de España ${ }^{28}$ (figura $n^{\circ} 6$ ).

\footnotetext{
${ }^{28}$ Este proyecto comenzó bajo los últimos días del mandato de José Luis Rodríguez Zapatero y se desarrolló en las primeras semanas del Gobierno de Mariano Rajoy. Las opiniones individuales -salvo en el caso de un experto que entendió que el cambio presidencial podía influir parcialmente en su caracterización informativa-, se centraron en el análisis de la presidencia del Gobierno como institución contemporánea y no en la personalización de su caracterización.
} 


\begin{tabular}{|c|c|c|c|c|c|c|}
\hline \multicolumn{3}{|c|}{ Espectro negativo } & \multirow[t]{2}{*}{ Espectro neutro } & \multicolumn{3}{|c|}{ Espectro positivo } \\
\hline $\begin{array}{l}\text { Extremada- } \\
\text { mente }\end{array}$ & Bastante & Poco & & Poco & Bastante & $\begin{array}{l}\text { Extremada- } \\
\text { mente }\end{array}$ \\
\hline & & & & & Abundante & \\
\hline & & & $\begin{array}{l}\text { Ni aburrida } \\
\text { ni interesante }\end{array}$ & & & \\
\hline & & & $\begin{array}{l}\text { Ni obsoleta } \\
\text { ni moderna }\end{array}$ & & & \\
\hline & & & $\begin{array}{l}\mathrm{Ni} \text { insignificante } \\
\text { ni importante }\end{array}$ & & & \\
\hline & & Opaca & & & & \\
\hline & & & Ni inútil ni útil & & & \\
\hline & Tendenciosa & & & & & \\
\hline & & & $\begin{array}{l}\text { Ni tabú ni } \\
\text { no tabú }\end{array}$ & & & \\
\hline & Direccional & & & & & \\
\hline & & & & Persuasiva & & \\
\hline
\end{tabular}

Fuente: elaboración propia

En este segundo caso asimismo se registró un alto nivel de consenso entre las opiniones individuales ${ }^{29}$. Según puede observarse en la figura $n^{\circ} 6$ apareció en el grupo una tendencia central mayoritaria a través de la cual los integrantes del plantel posicionaron la mayor parte de los adjetivos en el espectro neutro $(n=6)$, tres de ellos en el espectro negativo y dos adjetivos en el espectro positivo. La preferencia grupal por los adjetivos emplazados en el espectro neutro (a pesar de que en las instrucciones iniciales se pidió a los entrevistados que preferiblemente no valoraran los ítems en el espacio intermedio a menos que fuera indispensable), señala la libertad de que disponen los medios españoles para informar sobre el Presidente del Gobierno: sus noticias vienen definidas por valores internos tales como la política editorial, la tradición, el público objetivo, etcétera. En ese sentido, contrasta, por ejemplo, que los expertos hayan calificado como "bastante tendenciosa" la información presidencial frente a la valoración de "poco tendenciosa" de la información real, un hecho que tiene que ver con la compartición tanto de unas fuentes comunes como de un contexto simbólico en el caso de las noticias relativas a la Casa Real, lo que restringe el movimiento o parcialidad de cada medio. Los expertos asimismo reconocieron la inexistencia de un tabú informativo sobre la información presidencial, aunque sí que remarcaron su existencia en el caso de la información protagonizada por el monarca. Como elementos diferenciadores además de "tabú" el

${ }^{29}$ Para una información más detallada ver el Anexo 2 de este trabajo. 
grupo subrayó el carácter "obsoleto, aburrido, insignificante" e "inútil"30 de los tratamientos informativos alusivos al rey Juan Carlos en los medios españoles.

\subsection{Segundo bloque: valoraciones íntimas sobre las restricciones informativas de la monarquía}

Con este segundo bloque pretendíamos averiguar la valoración íntima de los entrevistados sobre la idoneidad de asumir una mayor transparencia informativa 0 de reconfigurar el blindaje simbólico que vertebra a la Casa Real. Como puede verse en la figura $n^{\circ} 7$ los expertos alcanzaron un alto nivel de acuerdo (con un $83,3 \%$ de las respuestas decantadas hacia uno de los extremos) sobre el reconocimiento de que existe una demanda social generalizada de una mayor transparencia informativa en los asuntos del Rey.

\section{Figura $n^{\circ}$ 7. Transparencia informativa sobre el rey Juan Carlos I}

\begin{tabular}{|l|c|}
\hline Dígame, por favor, ¿con quién está más de acuerdo? & Resultados \\
\hline $\begin{array}{l}\text { SEÑOR RODRÍGUEZ - Yo creo que los ciudadanos demandan una mayor transparencia en } \\
\text { los asuntos del Rey de España. }\end{array}$ & $83,3 \%$ \\
\hline $\begin{array}{l}\text { SEÑOR PÉREZ - No estoy de acuerdo. En mi opinión actualmente la información disponible } \\
\text { sobre el Rey es la adecuada. }\end{array}$ & $16,7 \%$ \\
\hline
\end{tabular}

Fuente: elaboración propia

En la siguiente pregunta, sin embargo, el grupo registró un importante desacuerdo al ser cuestionado sobre la conveniencia de desbloquear el blindaje informativo que rodea al monarca alrededor de determinadas cuestiones.

\section{Figura $n^{\circ}$ 8. Blindaje informativo del rey Juan Carlos I}

\begin{tabular}{l|c|}
\hline Dígame, por favor, ¿con quién está más de acuerdo? & Resultados \\
\hline $\begin{array}{l}\text { SEÑOR PÉREZ - Es necesario el blindaje informativo de la figura del jefe del Estado, porque } \\
\text { determinadas informaciones podrían motivar una inestabilidad política en el país. }\end{array}$ & $50 \%$ \\
\hline $\begin{array}{l}\text { SEÑOR RODRÍGUEZ - No estoy de acuerdo. Los medios de comunicación no deben hacer } \\
\text { distinciones informativas ni siquiera ante la jefatura del Estado: a mayor responsabilidad, } \\
\text { mayor transparencia. }\end{array}$ & $50 \%$ \\
\hline
\end{tabular}

Fuente: elaboración propia

\footnotetext{
${ }^{30}$ En términos de prestigio.
} 
Esta divergencia refleja la contradicción inherente a la naturaleza interna de los tabús informativos. Gantz, en función de sus características operativas, diferenció a los tabús como protectores y como destructores ${ }^{31}$. Esa doble vertiente aparece asimismo inextricablemente ligada al tabú de la Casa Real: sí hace falta -según los expertos-, una mayor transparencia informativa, pero también hace falta -y aquí surge el desacuerdo-, definir unos límites (o blindaje) a partir de los cuales los medios deben reconfigurar el espacio simbólico propio de la monarquía. Porque a diferencia de otras instituciones, la Casa Real es uno de los vectores que cohesionan de forma permanente al Estado, por lo que su inestabilidad puede generar resultados incontrolados en otras esferas tales como la económica, la social, la cultural o la política.

\subsection{Tercer bloque: creación de un nuevo espacio informativo entre la Casa Real y los medios}

A través del tercer bloque de preguntas pretendíamos sentar las bases para reconfigurar el espacio informativo que comparten la Casa Real y los medios de comunicación españoles. En la primera de las preguntas los expertos manifestaron un alto grado de acuerdo y consensuaron el hecho de que las restricciones informativas actuales perjudican a la reputación institucional del Rey. Sólo uno de los expertos se decantó por la tercera opción posible, "otros", e indicó que dichas restricciones ni perjudican ni benefician a la reputación real porque en su opinión forman parte de una estrategia que se administra en función de las necesidades del momento.

\begin{tabular}{|l|l|}
\hline \multicolumn{2}{|c|}{\begin{tabular}{l} 
Figura $\mathbf{n}^{\circ} 9$. \\
\multicolumn{2}{|c|}{ Efectos de las restricciones informativas en la reputación } \\
de la institución monárquica
\end{tabular}} \\
\hline $\begin{array}{l}\text { ¿Las restricciones informativas sobre el rey Juan Carlos benefician o perjudican a su } \\
\text { reputación institucional? }\end{array}$ & Resultados \\
\hline Benefician & $00,0 \%$ \\
\hline Perjudican & $83,3 \%$ \\
\hline Otros & $16,7 \%$ \\
\hline
\end{tabular}

Fuente: elaboración propia

En la primera ronda de entrevistas propusimos algunos tratamientos informativos novedosos que podían reforzar la caracterización mediática del Rey. Los expertos, además de valorarlos, aportaron individualmente algunas de las medidas. En la segunda ronda los integrantes del grupo tuvieron la oportunidad de puntuar estas medidas con el fin de establecer un criterio común. Conviene destacar la honestidad de algunos de los expertos que valoraron las aportaciones de sus colegas con puntuaciones más altas que las propias. La siguiente tabla recoge los tratamientos valorados en función de una escala

\footnotetext{
31 GANTZ, W., "The Movement of Taboos: A Message-Oriented Approach", Paper presented at the 61 $1^{\text {st }}$ Annual Meeting of the Association for Education in Journalism, U. S. Department of Health, Education and Welfare National Instituted of Education, Washington, 1978, p. 1.
} 
gradual de 1 a 5 puntos en donde 5 representaba la opción "muy importante" mientras que 1 codificaba la opción "nada importante"32.

\begin{tabular}{|c|c|c|c|c|}
\hline \multicolumn{5}{|c|}{$\begin{array}{c}\text { Figura } n^{\circ} \text { 10. Tratamientos informativos novedosos para reforzar } \\
\text { la agenda del Rey Juan Carlos }\end{array}$} \\
\hline & Media & Mediana & Moda $\mid$ & $\begin{array}{l}\text { Desv. } \\
\text { Típica }\end{array}$ \\
\hline Transparencia sobre las finanzas personales del Rey & 5,00 & 5,00 & 5 & 0 \\
\hline Previsiones sucesorias & 4,67 & 5,00 & 5 & 0,51 \\
\hline Información de la actividad del Rey & 4,33 & 5,00 & 5 & 1,21 \\
\hline Origen de sus bienes & 4,20 & 4,00 & 4 & 0,83 \\
\hline Perfil humano del monarca (aficiones, amistades, etc.) & 3,67 & 3,50 & 3 & 0,81 \\
\hline Declaraciones espontáneas a los medios & 3,50 & 3,50 & 2 & 1,37 \\
\hline $\begin{array}{l}\text { Intervenciones y posiciones políticas reales del Rey en los asuntos del } \\
\text { Estado }\end{array}$ & 3,50 & 3,50 & 3 & 1,51 \\
\hline Detalles sobre los viajes privados & 3,33 & 3,00 & 2 & 1,36 \\
\hline Preferencias culturales del monarca & 3,17 & 3,00 & 3 & 1,32 \\
\hline Origen familiar & 3,00 & 3,00 & 1 & 1,58 \\
\hline Vida íntima & 2,33 & 2,00 & 1 & 1,50 \\
\hline
\end{tabular}

Fuente: elaboración propia

Como puede observarse en 9 de los 11 ítems se registró una escasa desviación típica entre las opiniones individuales lo que confirmó un alto grado de consenso grupal en la mayor parte de los tratamientos propuestos. El ítem la "transparencia sobre las finanzas personales del Rey" se definió como "muy importante" $(n=5,00)$, con un consenso total del $100 \%$ de los expertos. En un segundo tramo el grupo situó las "previsiones sucesorias" $(n=4,67)$, la "información de la actividad del Rey" ( $n=4,33)$ y a pesar de la abstención de un experto también se incluyó dentro del tramo "bastante importante" el ítem el "Origen de sus bienes" (n=4,20). Estos ítems, que recibieron las mayores puntuaciones, hacían referencia a la necesidad de una mayor transparencia del discurso informativo de la Casa Real. Los dos ítems que registraron un nivel medio de consenso fueron, en primer lugar, "intervenciones y posiciones políticas reales del Rey en los asuntos del Estado" (n=3,50) el cual aludía a la posibilidad de que el monarca emitiera sus opiniones acerca de algunos temas concretos, por ejemplo, tal y como ya hace una vez al año a través de su tradicional mensaje navideño. Y en segundo lugar el ítem "origen familiar" (n=3,00) que contó con una abstención y que generó un nivel próximo al descuerdo. Esta controversia reflejaba las tendencias internas del grupo: la disconformidad en algunos ítems realzaba el valor de los ítems en que se había logrado un alto nivel de consenso ya que la divergencia recalcaba, por un lado, la diversidad ideológica del grupo y por el otro la precisión de las respuestas individuales que permitía descartar con seguridad el llamado efecto halo.

\footnotetext{
${ }^{32}$ Los dos ítems en cursiva representan la abstención de uno de los expertos en la votación grupal.
} 
En último lugar los expertos posicionaron la galería temática relativa a la "vida íntima" del Rey $(n=2,17)$, dentro del tramo "poco importante". Como se ha visto, en términos generales, para reforzar el prestigio de la institución el grupo incidió en un incremento de los temas puramente de gestión comunicacional antes que en los temas clasificados dentro del ámbito personal o privado del rey Juan Carlos.

Por último, se cuestionó a los expertos sobre la habilitación de algunas medidas interactivas que posibilitasen la reconfiguración del espacio relacional entre la Casa Real y los medios de comunicación españoles. Durante la primera ronda, de igual forma que en el apartado anterior, además de valorar los ítems propuestos los miembros del grupo aportaron algunos ítems nuevos que puntuaron colectivamente en la segunda ronda:

\begin{tabular}{|c|c|c|c|c|}
\hline \multicolumn{5}{|c|}{$\begin{array}{c}\text { Figura } n^{\circ} 11 . \text { Canales interactivos para reconfigurar el espacio relacional } \\
\text { entre la Casa del Rey y los medios }\end{array}$} \\
\hline & Media & Mediana & Moda & $\begin{array}{l}\text { Desv. } \\
\text { Típica }\end{array}$ \\
\hline $\begin{array}{l}\text { Transparencia en la gestión de la comunicación, como se le pide a } \\
\text { cualquier gabinete de comunicación }\end{array}$ & 4,67 & 5,00 & 5 & 0,51 \\
\hline Desaparición del secretismo con el que actúa la Casa del Rey & 4,50 & 4,50 & 4 & 0,54 \\
\hline Liberalización del tratamiento informativo al estilo británico & 4,33 & 4,50 & 5 & 0,81 \\
\hline Desclasificación de documentos históricos & 4,17 & 4,50 & 5 & 0,98 \\
\hline $\begin{array}{l}\text { Reconocimiento de que está pendiente un debate nacional sobre la } \\
\text { monarquía }\end{array}$ & 4,17 & 5,00 & 5 & 1,60 \\
\hline Posibilidad de que los periodistas realicen preguntas no pactadas & 3,83 & 3,50 & 3 & 0,98 \\
\hline $\begin{array}{l}\text { Creación de un manual que homogenice el protocolo de alusiones y } \\
\text { tratamientos al Rey }\end{array}$ & 3,50 & 3,00 & 3 & 1,22 \\
\hline Entrevistas ocasionales & 3,33 & 3,00 & 3 & 1,03 \\
\hline $\begin{array}{l}\text { Una política de información pactada con los principales diarios de } \\
\text { información general (exclusivas, entrevistas...) del país, sin } \\
\text { discriminación y rotatoria, de manera regular }\end{array}$ & 3,33 & 3,50 & 5 & 1,63 \\
\hline Habilitación de canales interactivos en la web oficial (email, chat...) & 3,17 & 3,00 & 2 & 1,16 \\
\hline
\end{tabular}

Fuente: elaboración propia

A diferencia de las medidas clasificadas dentro de la figura $n^{\circ} 10$ en este caso ninguno de los ítems recibió una puntuación equivalente a un consenso total, si bien 8 de los 10 ítems obtuvieron una escasa desviación típica lo que evidenciaba el alto grado de acuerdo entre las opiniones individuales. Los expertos calificaron dentro de la frecuencia "bastante importante" la "transparencia en la gestión de la comunicación" (n=4,67), la "desaparición del secretismo con el que actúa la Casa del Rey" ( $n=4,50)$, la "liberalización del tratamiento informativo al estilo británico" (n=4,33) y la "desclasificación de documentos históricos" $(n=4,17)$. El "reconocimiento de que está pendiente un debate nacional sobre la monarquía" $(n=4,17)$ registró un nivel medio de acuerdo así como el ítem "una política de información pactada con los principales diarios de información general" $(n=3,33)$. Los ítems que aludían a la posibilidad de que el monarca concediese entrevistas regularmente o que los periodistas realizasen preguntas no pactadas fueron incluidos 
dentro de la frecuencia intermedia, quizá porque los expertos entendieron que las declaraciones espontáneas del rey Juan Carlos pueden fomentar una inestabilidad estatal por la alteración del arbitraje simbólico que representa el monarca. En último término, dentro del tramo calificado como "importante", el grupo puntuó la "habilitación de canales interactivos en la web oficial" (n=3,17). En las puntuaciones grupales ningún ítem mereció una valoración inferior a los 3 puntos, es decir, ninguna de las medidas propuestas se clasificó en los escalones tipificados como "poco" o "nada importante". A pesar de que dos de las medidas enfrentaron a los miembros del grupo, la tendencia generalizada apuntaba a una necesidad de la institución monárquica de reforzar su gestión informativa.

\section{Conclusiones}

Si la percepción que los ciudadanos construyen sobre la monarquía viene en su mayor parte influenciada por la caracterización informativa de su primer representante, es decir, del rey Juan Carlos, en este trabajo la opinión grupal ha sido concluyente al respecto al definir dicha caracterización como "aburrida, obsoleta, insignificante, inútil, tendenciosa" y sobre todo "opaca, tabú, direccional". La caída de la popularidad de la monarquía incluso antes de ese fenómeno judicial y mediático conocido como caso Urdangarin-, tiene que ver con el anclaje comunicacional de la institución en un contexto dominado por un incremento de la interactividad o feedback social. Dentro de ese paradigma participativo contemporáneo la alta concentración de restricciones, según los expertos, perjudica a la reputación de la institución monárquica porque la sitúa en un nivel comunicativo alejado de las expectativas de los ciudadanos. Este trabajo, además de presentar las respuestas concretas de un grupo de expertos altamente especializados, pretende fomentar el debate sobre las medidas que ha de adoptar la Casa Real para dinamizar su discurso informativo. Una dinamización que ha de integrar la revisión de sus elementos pancomunicacionales entre los cuales, con Sierra Sánchez, incluimos al protocolo ${ }^{33}$. La Casa Real ya es consciente de esa necesaria actualización: el desglose presupuestario, mencionado en la introducción, representa una de las medidas más importantes adoptadas por la institución en los últimos treinta y seis años ${ }^{34}$. Creemos que una de las soluciones del problema, tal y como se deduce de las opiniones recabadas, pasa por la reconfiguración del espacio informativo, o sea de los límites asumidos que comparten la Casa Real y los medios. Por un lado la información sobre el Rey debe garantizar la estabilidad simbólica de la institución como factor de convivencia (articulando, por ejemplo, un tabú sobre la vida íntima del monarca), y por el otro debe estimular la armonización con el discurso social a través de los ejes de:

\footnotetext{
33 Cfr. SIERRA SÁNCHEZ, J., "Protocolo: herramienta comunicativa persuasiva y simbólica", ZER: Revista de Estudios de Comunicación, núm. 24, 2008, pp. 337-361.

${ }^{34}$ Asimismo, entre 2011 y 2013 la Casa Real organizó multitud de medidas comunicacionales para aproximarse a la sociedad española, como comentamos en BARREDO IBÁÑEZ, D., El tabú Real. La imagen de una monarquía en crisis, 2013, pp. 27 - 33.
} 
1. Una adecuación a las diversas demandas de transparencia:

1.1 Transparencia financiera. Los expertos recomiendan a la Casa Real redefinir una mayor transparencia de las finanzas personales del Rey con un detallado desglose de los gastos ${ }^{35}$, propiedades inmobiliarias 0 de activos financieros, pago de impuestos, donaciones o regalos de otros líderes extranjeros, etcétera.

1.2 Transparencia institucional. Asimismo la opinión grupal propone una mayor transparencia de la gestión informativa de la institución. En esta esfera se plantea la instauración de una política de información pactada con los principales medios de información general, sin discriminación en función del sesgo ideológico y de manera regular y rotatoria. Dentro de esa política de información podrían incluirse como medidas internas las entrevistas exclusivas al rey o la posibilidad de que los periodistas realizasen preguntas no pactadas, con la consiguiente publicación de las posiciones y las opiniones del monarca ante determinados conflictos o asuntos del Estado.

2. La interactividad con la sociedad a la que representa mediante:

\subsection{Liberalización informativa:}

2.1.1 Histórica. Con la desclasificación de documentos reservados sobre las reuniones del monarca con otros jefes de Estado o personalidades o su participación en episodios históricos.

2.1.2 Contemporánea. Con una reducción de la textura restrictiva siguiendo el ejemplo del modelo británico y eliminando la alta concentración de elementos simbólicos arbitrarios característicos de la monarquía española. Se sugiere una mayor exposición del perfil humano del rey (con una paulatina reorientación de los estereotipos forjados por los medios), una descripción de sus viajes privados, así como la aclaración sobre las previsiones sucesorias.

Trabajando sobre estos vectores puede evitarse, en definitiva, la fosilización del entorno informativo de la Casa Real en el contexto comunicacional español.

\footnotetext{
${ }^{35}$ En el Capítulo VI del desglose presupuestario de 2011 se indicaba, por ejemplo, que las inversiones reales en equipos y derivados informáticos ascendía a $100.000 €$, pero no se especificaba cómo se distribuía esa partida. Para más información v. nota al pie número 10 de este trabajo.
} 


\section{BIBLIOGRAFÍA}

ARCE FERNÁNDEZ, C., STEM, D. E., ANDRADE FERNÁNDEZ, E. M. y SEOANE PESQUEIRA, G., "Comparación de escalas de respuesta para la medición de las actitudes", Psicothema, núm. 9, vol. 3, 1997, pp. 541-545.

BARREDO IBÁÑEZ, D., "Tabús informativos individuales y organizacionales del periodismo español en el siglo XXI: un estudio Delphi", Fonseca, Journal of Communication, núm. 3, 2011 , pp. $97-130$.

BARREDO IBÁÑEZ, D., El tabú de la expresividad real. Análisis del tratamiento informativo del rey Juan Carlos I en ABC.es y ELPAís.com (2009 - 2011) <tesis doctoral>, Málaga, Universidad de Málaga, 2012.

BARREDO IBÁÑEZ, D., El tabú Real. La imagen de una monarquía en crisis, Córdoba, Berenice, 2013.

BECOÑA IGLESIAS, E., "El Diferencial Semántico en la medición de actitudes: adjetivos versus conceptos, Revista de Psicología General y Aplicada, núm. 43, vol. 3, 1990, pp. 301 312.

EUROPEAN COURT OF HUMAN RIGHTS, "An elected representative's conviction for causing serious insult to the King of Spain was contrary to his freedom of expression", Press release by the Registrar of the Court, núm. 215, 2011, pp. 1-5.

GANTZ, W., "The Movement of Taboos: A Message-Oriented Approach", Paper presented at the $61^{\text {st }}$ Annual Meeting of the Association for Education in Journalism, U. S. Department of Health, Education and Welfare National Instituted of Education, Washington, 1978.

LANDETA, J., El método Delphi. Una técnica de previsión para la certidumbre, Ariel, Barcelona, 1999.

LINSTONE, H. A. y TUROFF, M., "Introduction", en LINSTONE, H. A. y TUROFF, M. (eds.), The Delphi method: Techniques and applications, Addison-Wesley Publishing Company, Reading, 1975, pp. 3-12.

LÓPEZ - ESCOBAR, E., LLAMAS, J. y MCCOMBS, M., "Una dimensión social de los efectos de los medios de difusión: agenda-setting y consenso", Comunicación y Sociedad, núms. 1 y 2, 1996, pp. $91-125$.

NADELSTICHER, A., Técnicas para la construcción de cuestionarios de actitudes y opción múltiple, Instituto Nacional de Ciencias Penales, México, 1983.

NOELLE - NEUMANN, E., La espiral del silencio. Opinión pública: nuestra piel social, Paidós, Barcelona, 1995. 
OSGOOD, C. E., SUCl, G. J. y TANNENBAUM, P. H., The measurement of meaning, University of Illinois Press, Illinois, 1957.

RAMOS, F., "El secuestro de El Jueves y las injurias periodísticas a la Corona, un injustificable ataque a la libertad de expresión", ÁMBITOS, núm. 16, 2007, pp. 151 - 186.

ROWE, G. y WRIGHT, G., "The Delphi technique as a forecasting tool: issues and analysis", International Journal of Forecasting, núm. 15, 1999, pp. 353-375.

SIERRA SÁNCHEZ, J., "Protocolo: herramienta comunicativa persuasiva y simbólica", ZER: Revista de Estudios de Comunicación, núm. 24, 2008, pp. 337-361.

SKULMOSKI, G., HARTMAN, F. y KRAHN, J., "The Delphi Method for Graduate Research", Journal of Information Technology Education, núm. 6, 2007, pp. 1-21.

TEZANOS, J. F., "El método Delphi y la anticipación de escenarios y tendencias socio políticas", Sistema: revista de ciencias sociales, núm. 213, 2009, pp. 83-104.

YOUNG, S. J. y JAMIESON, L. M., "Delivery methodology of the Delphi: a comparison of two approaches", Journal of Park and Recreation Administration, núm. 19, vol. 1, 2001, pp. 4258.

ZUGASTI, R., La forja de una complicidad. Monarquía y Prensa en la Transición española (1975 - 1978), Fragua, Madrid, 2007.

ZUGASTI, R., "La prensa española de la transición como escenario de apoyo político a Juan Carlos I: el ejemplo de la legitimidad dinástica de la monarquía", Palabra Clave, núm. 10, vol. 1, 2007, pp. 60-70.

ZUGASTI, R., "El papel de la prensa en la construcción de la democracia española: de la muerte de Franco a la Constitución de 1978", Confines, 4/7, enero-mayo, 2008, pp. 53-68.

\section{WEBGRAFÍA}

CIS, "Barómetro de octubre", núm. 2914, 2011, obtenido el 10 de enero de 2012 de: http://www.cis.es/cis/export/sites/default/-

Archivos/Marginales/2900_2919/2914/Es2914.pdf.

DE BORBÓN, J. C., "Mensaje de Navidad de Su Majestad el Rey", Casa de Su Majestad el Rey, 24-12-2011, obtenido el 10 de enero de 2012 de:

http://www.casareal.es/noticias/news/20111224_mensaje_navidad-ides-idweb.html.

HSU, C.-C. y SANDFORD, B. A., "The Delphi Technique: Making Sense of Consensus", Practical Assesment Research and Evaluation, núm. 12, vol. 10, 2007, obtenido el 10 de enero de 2012 de: http://pareonline.net/pdf/v12n10.pdf. 
MANRESA, A., "El juez levanta el secreto e imputa a Iñaki Urdangarin", El País, 29-12-2011, obtenido el 10 de enero de 2012 de:

http://politica.elpais.com/politica/2011/12/29/actualidad/1325146927_909959.html.

CASA REAL, "Presupuesto anual", Casa de Su Majestad el Rey, (sin fecha), obtenido el 10 de enero de 2012 de: http://www.casareal.es/laCasa/presupuesto-ides-idweb.html.

\section{ANEXOS}

Anexo $n^{\circ} 1$. Estadísticas grupales sobre la caracterización informativa del rey Juan Carlos

Estadísticas grupales sobre la caracterización informativa del Rey Juan Carlos

\begin{tabular}{|l|c|c|c|c|}
\hline & Media & Mediana & Moda & Desv. Típica \\
\hline $\begin{array}{l}\text { Escasa (1) } \\
\text { Abundante (7) }\end{array}$ & 4,67 & 5,00 & 5 & 1,82 \\
\hline $\begin{array}{l}\text { Aburrida (1) } \\
\text { Interesante (7) }\end{array}$ & 3,67 & 3,50 & 1 & 2,16 \\
\hline $\begin{array}{l}\text { Obsoleta (1) } \\
\text { Moderna (7) }\end{array}$ & 3,33 & 3,00 & 2 & 1,50 \\
\hline $\begin{array}{l}\text { Insignificante (1) } \\
\text { Importante (7) }\end{array}$ & 3,83 & 3,50 & 2 & 1,94 \\
\hline $\begin{array}{l}\text { Opaca (1) } \\
\text { Transparente (7) }\end{array}$ & 2,50 & 2,00 & 2 & 2,25 \\
\hline $\begin{array}{l}\text { Inútil (1) } \\
\text { Útil (7) }\end{array}$ & 3,17 & 2,50 & 2 & 1,94 \\
\hline $\begin{array}{l}\text { Tendenciosa (1) } \\
\text { Equilibrada (7) }\end{array}$ & 3,67 & 3,00 & 3 & 2,33 \\
\hline $\begin{array}{l}\text { Tabú (1) } \\
\text { No tabú (7) }\end{array}$ & 2,50 & 2,00 & 1 & 1,87 \\
\hline $\begin{array}{l}\text { Direccional (1) } \\
\text { Interactiva (7) }\end{array}$ & 2,00 & 1,00 & 1 & 2,00 \\
\hline $\begin{array}{l}\text { Disuasiva (1) } \\
\text { Persuasiva (7) }\end{array}$ & 4,00 & 3,50 & 3 & 1,78 \\
\hline Fuente: Elaboracion & propia & & & \\
\hline
\end{tabular}

Fuente: Elaboración propia 
Anexo $n^{\circ} 2$. estadísticas grupales sobre la caracterización informativa del Presidente del Gobierno

\begin{tabular}{|l|c|c|c|c|}
\hline \multicolumn{5}{|c|}{$\begin{array}{c}\text { Estadísticas grupales sobre la caracterización } \\
\text { informativa del Presidente del Gobierno }\end{array}$} \\
\hline & Media & Mediana & Moda & Desv. Típica \\
\hline $\begin{array}{l}\text { Escasa (1) } \\
\text { Abundante (7) }\end{array}$ & 6,33 & 7,00 & 7 & 1,03 \\
\hline $\begin{array}{l}\text { Aburrida (1) } \\
\text { Interesante (7) }\end{array}$ & 4,00 & 5,00 & 5 & 2,00 \\
\hline $\begin{array}{l}\text { Obsoleta (1) } \\
\text { Moderna (7) }\end{array}$ & 4,33 & 5,00 & 5 & 1,96 \\
\hline $\begin{array}{l}\text { Insignificante (1) } \\
\text { Importante (7) }\end{array}$ & 4,50 & 5,00 & 5 & 1,87 \\
\hline $\begin{array}{l}\text { Opaca (1) } \\
\text { Transparente (7) }\end{array}$ & 3,50 & 3,50 & 1 & 1,87 \\
\hline $\begin{array}{l}\text { Inútil (1) } \\
\text { Útil (7) }\end{array}$ & 4,33 & 5,00 & 5 & 1,96 \\
\hline $\begin{array}{l}\text { Tendenciosa (1) } \\
\text { Equilibrada (7) }\end{array}$ & 2,67 & 2,50 & 1 & 1,86 \\
\hline $\begin{array}{l}\text { Tabú (1) } \\
\text { No tabú (7) }\end{array}$ & 4,67 & 5,00 & 5 & 2,06 \\
\hline $\begin{array}{l}\text { Direccional (1) } \\
\text { Interactiva (7) }\end{array}$ & 2,33 & 1,00 & 1 & 2,06 \\
\hline $\begin{array}{l}\text { Disuasiva (1) } \\
\text { Persuasiva (7) }\end{array}$ & 5,17 & 5,50 & 3 & 1,83 \\
\hline Fuente: Eabocion & & & \\
\hline
\end{tabular}

Fuente: Elaboración propia 